\title{
4-methylesculetin, a coumarin derivative, ameliorates dextran sulfate sodium-induced intestinal inflammation
}

\author{
Aline Witaicenis ${ }^{\mathrm{a}, *}$, Ellen Cristina Souza de Oliveira ${ }^{\mathrm{a}}$, Alexandre Tanimoto ${ }^{\mathrm{a}}$, \\ Sofia Fernanda Gonçalves Zorzella-Pezavento ${ }^{\mathrm{b}}$, Silvio Luis de Oliveira ${ }^{\mathrm{b}}$, Alexandrina Sartori ${ }^{\mathrm{b}}$, \\ Luiz Claudio Di Stasi ${ }^{\mathrm{a}}$ \\ ${ }^{\text {a } U n i v e r s i d a d e ~ E s t a d u a l ~ P a u l i s t a ~(U N E S P), ~ I n s t i t u t e ~ o f ~ B i o s c i e n c e s, ~ D e p a r t m e n t ~ o f ~ P h a r m a c o l o g y, ~ C a ̂ m p u s ~ B o t u c a t u, ~ S P, ~ B r a z i l ~}$ \\ ${ }^{\mathrm{b}}$ Universidade Estadual Paulista (UNESP), Institute of Biosciences, Department of Microbiology and Immunology, Câmpus Botucatu, SP, Brazil
}

\section{A R T I C L E I N F O}

\section{Keywords:}

Inflammatory bowel disease

4-methylesculetin

Coumarin

DSS

Antioxidant

Mice

\begin{abstract}
A B S T R A C T
4-methylesculetin is one of the coumarin derivatives with great anti-oxidant and anti-inflammatory activities. Recent studies have shown that 4-methylesculetin has a promising potentiality to treat inflammatory diseases, especially those related to reactive oxygen species, as inflammatory bowel disease. Based on this, the present study aims to investigate the intestinal anti-inflammatory activity of 4-methylesculetin in dextran sulfate sodium (DSS) model. For this purpose, mice received DSS 5\% for 5 days followed by 2 days of filtered tap water. Treated groups received orally 5 or $25 \mathrm{mg} / \mathrm{kg}$ of 4-methylesculetin daily since the first day. Macroscopic, microscopic and biochemical parameters were evaluated. 4-methylesculetin $(25 \mathrm{mg} / \mathrm{kg})$ improved microscopic parameters, decreased MPO activity, reduced the colonic levels of IL- 6 and counteracted GSH depletion when compared with DSS-control group. Our results show the intestinal anti-inflammatory activity of 4-methylesculetin in DSS model, which is related to its antioxidant and anti-inflammatory properties. This way, 4-methylesculetin, is a new potential compound for treatment of both types of IBD.
\end{abstract}

\section{Introduction}

Coumarins comprise a very large class of phenolic substances, naturally found in plants or as synthetic compound, which are made of fused benzene and $\alpha$-pyrone rings, with different substitutes on the basic molecule of coumarin. These substitutions seem to define the diverse biological activities, such as, anti-inflammatory, antioxidant, antinociceptive, hepatoprotective, antithrombotic, antiviral, antimicrobial, antituberculosis, anti-carcinogenic, antidepressant, antihyperlipidemic and anticholinesterase [1,2].

4-methylesculetin (6,7-Dihydroxy-4-Methylcoumarin) is one of the coumarin derivatives with great anti-oxidant and anti-inflammatory activities [3-6]. This molecule has been shown non-toxic effects [7] and a promising potentiality to treat inflammatory diseases, especially those related to reactive oxygen species (ROS) $[5,6,8]$.

Inflammatory bowel disease (IBD) comprises mainly Crohn's disease (CD) and ulcerative colitis (UC), and both are characterized by chronic or relapsing immune activation and inflammation within the gastrointestinal tract. Several factors are believed to have a role in their development, including dysregulated immune responses, host genotype and environmental factors. Although etiology of IBD is not totally elucidated, several studies had focused on reactive oxygen species (ROS) as an important contributors to IBD development [9]. IBD patients show excessive oxidant activities and a decrease in antioxidant defenses. This excess of ROS reacts with cell membrane fatty acids, proteins and DNA permanently impairing their functions. These damage caused by ROS can be reversed by restored in the antioxidant balance [10]. Therefore, the administration of antioxidants compounds with additional anti-inflammatory action may be potential in the treatment of IBD [9].

Several studies have shown the beneficial effects of different natural antioxidant compounds in experimental models of intestinal inflammation, including flavonoids such as quercitrin [11], rutoside [12], morin [13], diosmin and hesperidin [14], isocoumarin [15] and

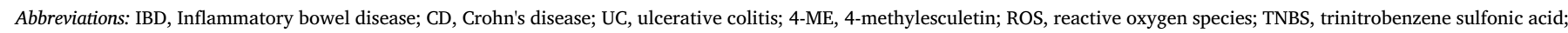

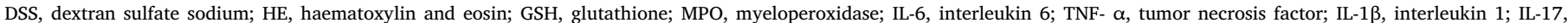
interleukin 17

* Corresponding author. Laboratory of Phytomedicines, Pharmacology and Biotechnology (PhytoPharmaTech), Department of Pharmacology, Institute of Biosciences, Universidade Estadual Paulista (UNESP), Botucatu, 18618-970, SP, Brazil.

E-mail address: aline.wit@ibb.unesp.br (A. Witaicenis). 


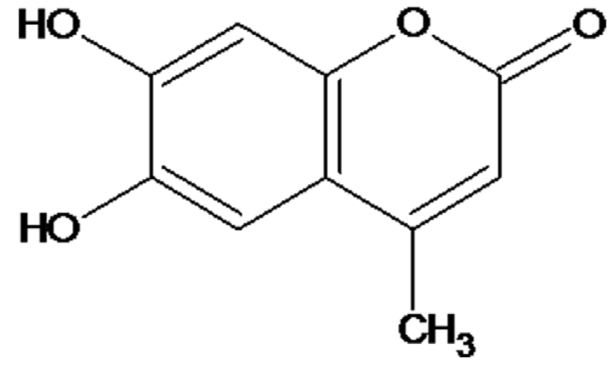

Fig. 1. Chemical structure of 4-methylesculetin (6,7-dihydroxy-4-methylcoumarin).

coumarin derivatives as 4-hydroxycoumarin, coumarin [5,16-18] esculetin, 4-methylesculetin $[5,6,17]$, scopoletin, scoparone, fraxetin, 4methylumbelliferone, esculin and daphnetin [18].

Based on this, the present study aims to investigate the intestinal anti-inflammatory activity of 4-methylesculetin in intestinal inflammation induced by DSS in mice.

\section{Material and methods}

\subsection{Material}

All chemicals, including 4-methylesculetin (Fig. 1) and dextran sulfate sodium (DSS) were purchased from Sigma Aldrich (São Paulo, Brazil). The tested substances were dissolved in methylcellulose (1\% w/ v) and were prepared daily and immediately before administration to the animals.

\subsection{Animals}

Male Swiss albino mice (25-35 g) obtained from Anilab laboratory, Paulinia - São Paulo State were housed in standard environmental conditions $\left(21{ }^{\circ} \mathrm{C}, 60-70 \%\right.$ humidity) with a $12 \mathrm{~h}$ light/dark cycle and air filtration. The animals had free access to water and food (Biobase, São Paulo, Brazil) until the beginning of the experimental design. The experimental protocols were conducted according to the "Guidelines of Animal Experimentation" approved by the Commission of Ethics in Animal Experimentation (Protocol number 2014/616-CEEA), Institute of Biosciences, Universidade Estadual Paulista (UNESP).

\subsection{Induction and assessment of the intestinal inflammatory process}

Mice received sterile, filtered water containing $5 \%(\mathrm{w} / \mathrm{v})$ DSS (40 kDa; Sigma) ad libitum for 5 days, followed by 2 days of regular filtered tap water. Oral administration of 4-ME or vehicle proceeded daily since the first day. The following parameters were daily monitored: food and DSS consumption, water intake, body weight, diarrhea and rectal bleeding. Animals from all groups $(n=8)$ were killed on the eighth day of experimentation by cervical dislocation.

The colonic segments were then obtained after laparotomy, placed on an ice-cold plate, cleaned of fat and mesentery tissue, and blotted on filter paper. The colon was weighted, and its length was measured under a constant load ( $2 \mathrm{~g}$ ) to the determination of colonic weight/ length ratio. The colon was subsequently divided longitudinally into different pieces to be used for the biochemical and histological analyses.

\subsection{Biochemical and immunological evaluation}

Myeloperoxidase (MPO) activity and colon glutathione (GSH) content were quantified by the methodologies described by Krawisz [19] and Anderson [20], respectively.

TNF- $\alpha$, IL-17 and IL- 6 levels were quantified by enzyme-linked immunosorbent assay (ELISA) in colonic samples using DuoSet Kits
(R\&D Systems, Inc., Minneapolis, Minnesota, USA) according to the manufacturer's instructions.

\subsection{Histological evaluation}

A representative colon fragment was collected for histological slide preparation and stained with haematoxylin and eosin (HE) for analysis of the microscopic damage and was adapted and assigned on a $0-22$ scale as described by Dieleman et al. [21], and Stucchi et al., [22]. Images were acquired using Zeiss Imager Axio Vision 4.8.2.0 software.

\subsection{Statistical analyses}

Parametric data are expressed in mean \pm S.E.M. and were analyzed by one way analysis of variance (ANOVA) and post hoc least significance tests. Nonparametric data are expressed as the median (range) and were analyzed by Kruskal-Wallis test and post hoc least significance tests. All statistical analyses were calculated by Graphpad Prism $6.01^{\circ}$ with $\mathrm{p}<0.05$ set as the lowest level of statistical significance.

\section{Results and discussion}

Administration of DSS in drinking water of rodents results in colonic inflammation and resembles clinical and histopathological characteristics of human UC [23]. The exact mechanism by which DSS induces intestinal inflammation is unknown, but a alteration of gut permeability has been suggested [24]. A concentration-dependent mechanism with direct cytotoxicity of DSS on colonocytes, which leads to alteration of integrin- $\alpha 4$ and M290 subunit levels on epithelial cells [25] disrupting their interaction with the $\gamma \delta$-intraepithelial T cells involved in mucosal protection [26] has also been proposed. The breakdown of the intestinal barrier defense was shown to increase invasion by antigens, leading to continuous immune system stimulation with recruitment and activation of inflammatory cells, upregulation of inflammatory mediators [23], increase in ROS and decrease in colonic antioxidant defense [27].

On the present study, mice exposure to DSS for five days developed an acute intestinal inflammatory response which was confirmed by clinical symptoms such as diarrhea and rectal bleeding. DSS also triggered an increase in inflammatory markers and histological alterations. Concerning clinical parameters, the DSS-control group presented decrease in body weight and in food intake compared with the non-colitic group; however treatments were not able to change these parameters (Fig. 2).

Histologically, the DSS-control group was characterized by severe inflammation, lesions throughout the mucosa, alteration of epithelial structure, increase in leucocyte infiltration into the mucosa and submucosa, edema, vascularization and loss of crypts when compared to the non-colitic group (Figs. 2D and 3). Compared to the DSS-control group, the animals treated with 4-ME at the dose of $25 \mathrm{mg} / \mathrm{kg}$ showed mild inflammation, areas of intact epithelium and fewer ulceration spots, minor crypt damage, edema, infiltration of polymorphonuclear cells in submucosal layer as show by Figs. 2D and 3. However the difference was not statistically significant, the treatment with 4-ME avoided the development of intestinal inflammatory process, these improvement in histological parameters were also confirmed by decreased colon weight/length ratio in 4-ME treated animals (Fig. 4A) [28].

The colonic inflammation was associated with increased MPO activity and IL-6 levels and depleted GSH content when compared to noncolitic animals (Fig. 4). 4-ME administration at the dose of $25 \mathrm{mg} / \mathrm{kg}$ reduced MPO activity (Fig. 4B), IL-6 levels (Fig. 4D) and counteracted GSH depletion (Fig. 4C) induced by inflammatory process.

MPO activity has been widely used as a biomarker of intestinal inflammation; therefore, reduction of MPO enzymatic activity can be also interpreted as a manifestation of anti-inflammatory property of a given 
A

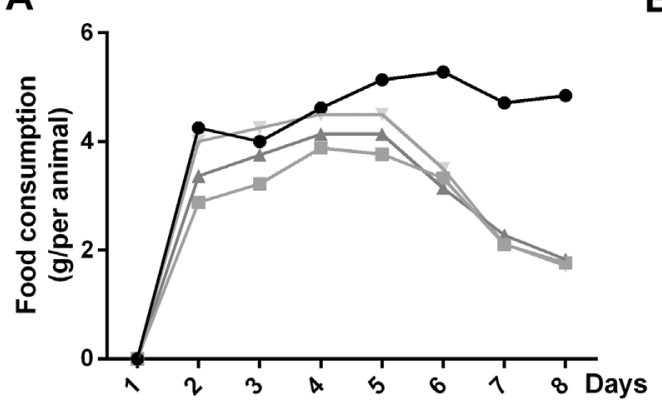

C

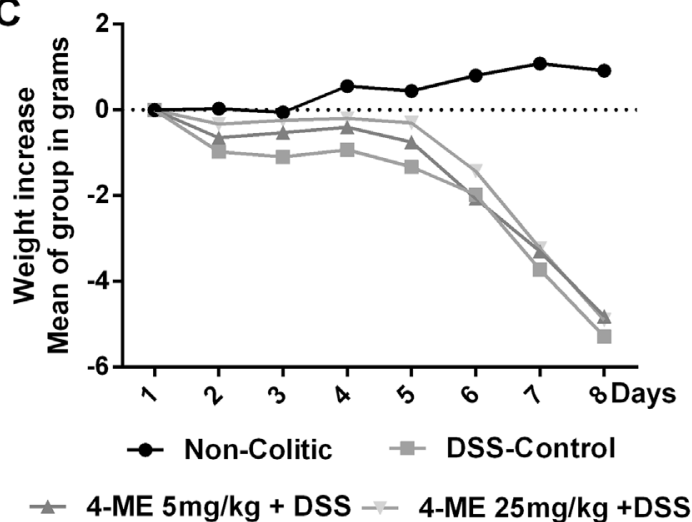

B

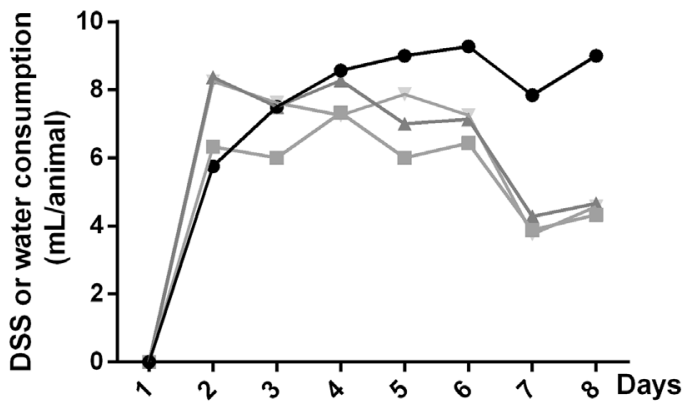

- Non-colitic $\square$ DSS-Control

D

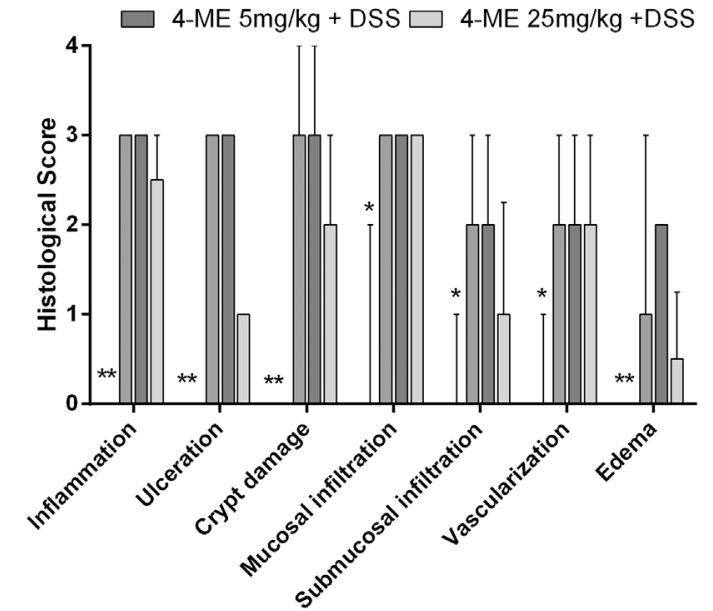

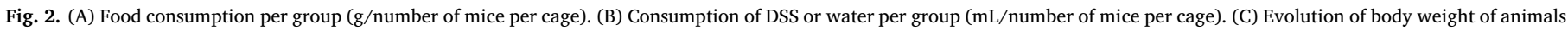

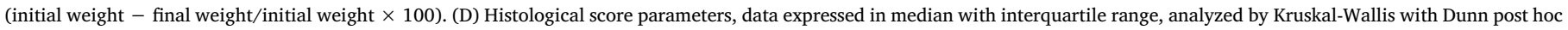
test, with ${ }^{*} \mathrm{p}<0.05$ and ${ }^{* *} \mathrm{p}<0.01$ compared to DSS-control $(\mathrm{n}=8)$.
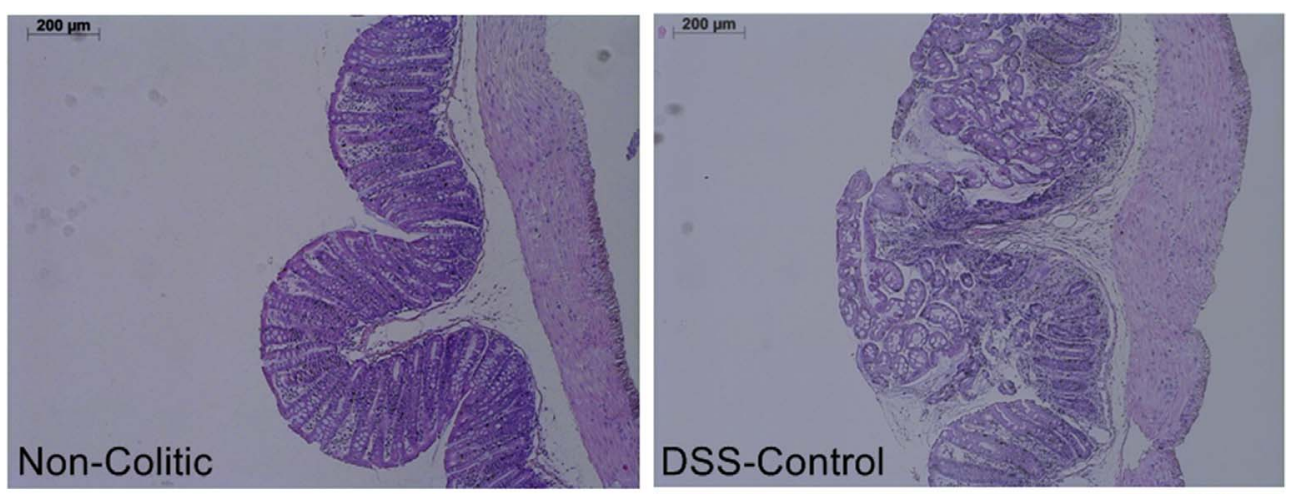

Fig. 3. Photomicrography of mice colon submitted to DSS-induced intestinal inflammation. Full thickness histological sections stained with hematoxylin and eosin.
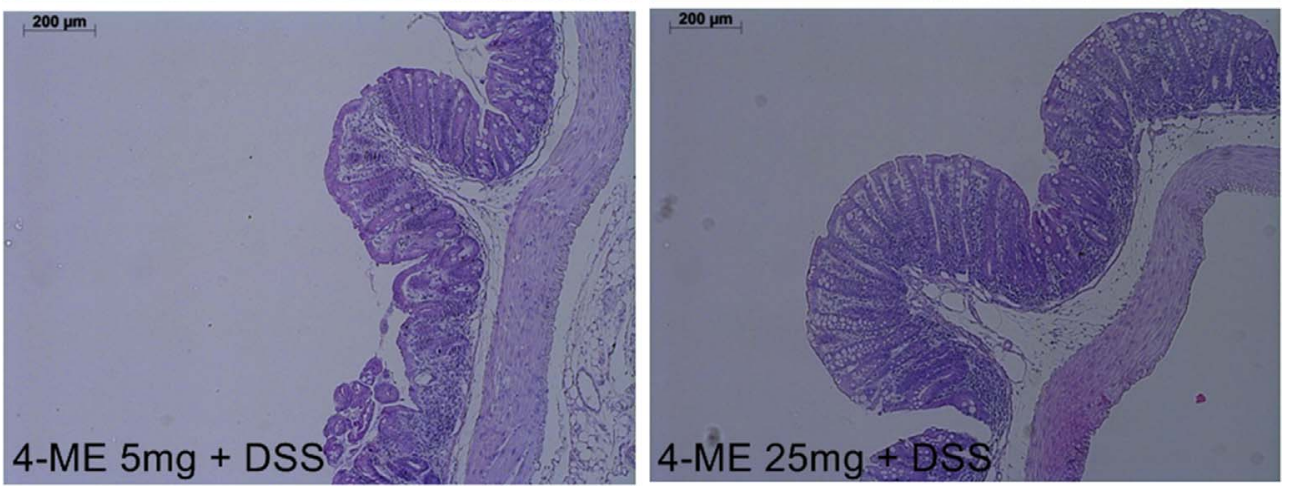

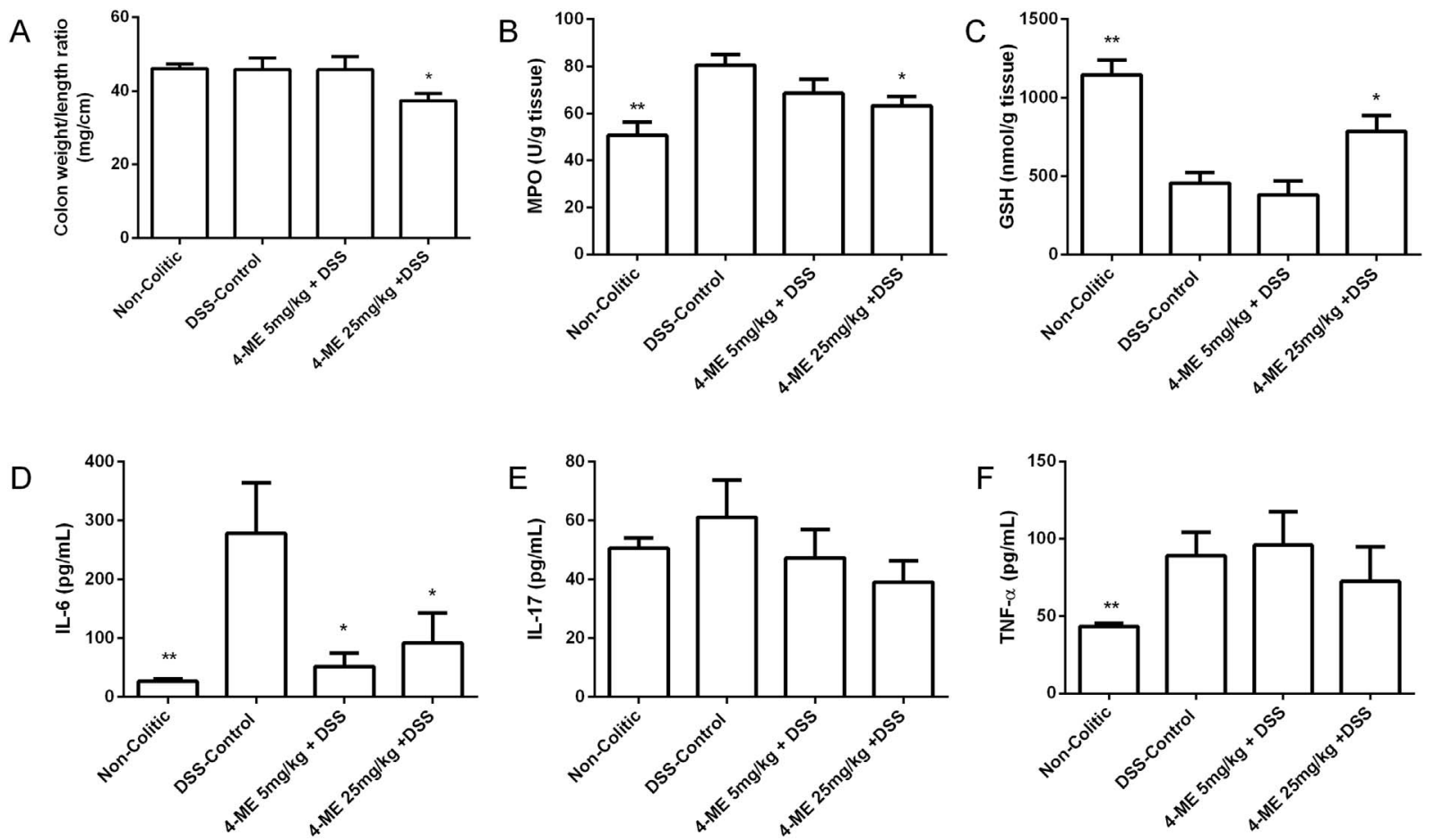

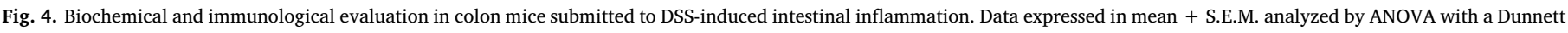
post hoc test, with ${ }^{*} \mathrm{p}<0.05$ and ${ }^{* *} \mathrm{p}<0.01$ compared to DSS-control $(\mathrm{n}=8)$.

compound [19]. Furthermore, MPO enzyme is responsible for the overproduction of reactive oxygen species, through catalyzation of hypochlorous acid production from hydrogen peroxide and chloride anion, consisting in one of the most cytotoxic compounds to bacteria and other pathogens [29]. GSH is the main non-enzymatic free-radical scavenger of toxic products of oxygen and other hydroperoxides. In oxidized form, GSH is easily diffuse out of the cell which leads to depletion in intracellular glutathione content, so the oxidative stress can also be measured by GSH levels [30]. Since MPO activity is directly proportional to neutrophils migration and it is also responsible for the reduction in the antioxidant defenses of the intestinal epithelia [31], the inhibition of MPO activity accompanied by GSH maintenance in treated animals, confirms the intestinal antioxidant and anti-inflammatory effects of 4-methylesculetin. Besides, 4-ME is the most potent inhibitory compound on the neutrophils oxidase metabolism, inhibiting MPO and elastase enzymes activities and acting as $\mathrm{HOCl}$ scavenger [32].

Several studies have demonstrated the antioxidant and anti-inflammatory properties of 4-ME. In the model of arthritis-induced oxidative stress 4-ME displayed an anti-inflammatory activity by restoring levels of superoxide dismutase, catalase, glutathione-S-transferase and GSH and total thiols [8]. In TNBS intestinal inflammation induced by TNBS in rats, 4-ME inhibited the MPO activity, reduced malondialdehyde colonic level, maintained the GSH content and ameliorated macro and microscopy parameters when administered at the dose at $5 \mathrm{mg} / \mathrm{kg}[5,6]$.

Therapeutic potential of 4-methylesculetin observed in this and in other studies could be attributed to its potent antioxidant and free radical scavenging properties previously described $[4-6,8]$. In intestinal inflammation, ROS production activated neutrophils and macrophages that exhibit massive intestinal mucosa infiltration with subsequent ROS release in large amounts. The enhanced production of reactive oxygen and nitrogen species is closely related to destructive effects on DNA, proteins and lipids, contributing to initiation and progression of intestinal inflammation with increased production of cytokines such as tumor necrosis factor (TNF- $\alpha$ ), interleukins IL- 6 and IL-1 $\beta[9,33]$.
Our results also showed the intestinal anti-inflammatory effect of 4ME was related to the inhibition of IL- 6 production, since treatments with 4-ME significantly reduced the levels of this cytokine when compared with DSS-control group (Fig. 4D). This is relevant because IL-6 plays a pivotal role in inflammation and a monoclonal antibody against IL-6 receptor has also been targeted in human IBD clinical trials with promising results [34].

Our results showed that 4-ME did not reduce the colonic TNF- $\alpha$ levels (Fig. 4F). Similar results were previously reported in the TNBS model [5], indicating that the intestinal anti-inflammatory activity of 4ME was not related to a reduction of TNF- $\alpha$ levels in the local of inflammation. In this experimental conditions the IL-17 levels were not altered in DSS-control group (Fig. 4E). In fact, studies showed that IL-17 is secreted by $\mathrm{Th} 17$ and $\mathrm{CD}^{+} \mathrm{T}$ cells during chronic inflammation, with its production increases only from thirteenth day after DSS administration [35].

No single model captures the complexity of human IBD, but each model provides valuable insights into one or another major aspect of disease [36]. The DSS (dextran sulfate sodium) model is characterized by Th1 polarization in earlier stage of disease and a mixed Th1/Th2 profile at chronic phase, resembling UC [37], while TNBS (2,4,6-trinitrobenzene sulfonic acid) model of intestinal inflammation has been considered closely related to Th1 response with several features similar to $\mathrm{CD}$. The use of both model represents a much more reliable experimental condition for evaluation of new potential drugs for the treatment of IBD. Based on these, we can suggest that 4-ME is a new promising compound for both types of IBD, UC and CD. However, further studies are still necessary for elucidating the mechanisms that could be involved in this intestinal anti-inflammatory effect.

\section{Conclusion}

The present study demonstrates, for the first time, the intestinal anti-inflammatory activity of 4-ME on DSS-induced intestinal inflammation in mice. Protective effects were related to antioxidant and anti-inflammatory properties of 4-ME. Furthermore, the intestinal anti- 
inflammatory activity provided by 4-ME highlights the relevance of this compound as a new complementary product for the treatment of both types of IBD.

\section{Acknowledgments}

This work was supported by FAPESP (São Paulo Research Foundation) with Grant Numbers 11/50512-2; 11/50824-4; 13/012977.

\section{References}

[1] S. Emami, S. Dadashpour, Current developments of coumarin-based anti-cancer agents in medicinal chemistry, Eur. J. Med. Chem. 102 (2015) 611-630.

[2] A.M. Katsori, D. Hadjipavlou-Litina, Coumarin derivatives: an updated patent review (2012-2014), Expert Opin. Ther. Pat. 24 (2014) 1323-1347.

[3] J.R. Hoult, M. Paya, Pharmacological and biochemical actions of simple coumarins: natural products with therapeutic potential, Gen. Pharmacol. 27 (1996) 713-722.

[4] M. Paya, B. Halliwell, J.R. Hoult, Interactions of a series of coumarins with reactive oxygen species. Scavenging of superoxide, hypochlorous acid and hydroxyl radicals, Biochem. Pharmacol. 44 (1992) 205-214.

[5] A. Witaicenis, A.C. Luchini, C.A. Hiruma-Lima, S.L. Felisbino, N. Garrido-Mesa, P. Utrilla, J. Galvez, L.C. Di Stasi, Suppression of TNBS-induced colitis in rats by 4methylesculetin, a natural coumarin: comparison with prednisolone and sulphasalazine, Chem. Biol. Interact. 195 (2012) 76-85.

[6] A. Witaicenis, L.N. Seito, L.C. Di Stasi, Intestinal anti-inflammatory activity of esculetin and 4-methylesculetin in the trinitrobenzenesulphonic acid model of rat colitis, Chem. Biol. Interact. 186 (2010) 211-218.

[7] E.L. Maistro, E. de Souza Marques, R.P. Fedato, F. Tolentino, A. da Silva Cde, M.S. Tsuboy, F.A. Resende, E.A. Varanda, In vitro assessment of mutagenic and genotoxic effects of coumarin derivatives 6,7-dihydroxycoumarin and 4-methylesculetin, J. Toxicol. Environ. Health A 78 (2015) 109-118.

[8] M. Hemshekhar, K. Sunitha, R.M. Thushara, M. Sebastin Santhosh, M. Shanmuga Sundaram, K. Kemparaju, K.S. Girish, Antiarthritic and antiinflammatory propensity of 4-methylesculetin, a coumarin derivative, Biochimie 95 (2013) $1326-1335$

[9] F.A. Moura, K.Q. de Andrade, J.C. Dos Santos, O.R. Araujo, M.O. Goulart, Antioxidant therapy for treatment of inflammatory bowel disease: does it work? Redox Biol. 6 (2015) 617-639.

[10] I.M. Balmus, A. Ciobica, A. Trifan, C. Stanciu, The implications of oxidative stress and antioxidant therapies in inflammatory bowel disease: clinical aspects and anmal models, Saudi J. Gastroenterol. 22 (2016) 3-17.

[11] F. Sanchez de Medina, B. Vera, J. Galvez, A. Zarzuelo, Effect of quercitrin on the early stages of hapten induced colonic inflammation in the rat, Life Sci. 70 (2002) 3097-3108.

[12] J. Galvez, T. Cruz, E. Crespo, M.A. Ocete, M.D. Lorente, F. Sanchez de Medina, A. Zarzuelo, Rutoside as mucosal protective in acetic acid-induced rat colitis, Planta Med. 63 (1997) 409-414.

[13] J. Galvez, G. Coelho, M.E. Crespo, T. Cruz, M.E. Rodriguez-Cabezas, A. Concha, M. Gonzalez, A. Zarzuelo, Intestinal anti-inflammatory activity of morin on chronic experimental colitis in the rat, Aliment. Pharmacol. Ther. 15 (2001) 2027-2039.

[14] M.E. Crespo, J. Galvez, T. Cruz, M.A. Ocete, A. Zarzuelo, Anti-inflammatory activity of diosmin and hesperidin in rat colitis induced by TNBS, Planta Med. 65 (1999) 651-653.

[15] L.C. Di Stasi, D. Camuesco, A. Nieto, W. Vilegas, A. Zarzuelo, J. Galvez, Intestinal anti-inflammatory activity of paepalantine, an isocoumarin isolated from the capitula of Paepalanthus bromelioides, in the trinitrobenzenesulphonic acid model of rat colitis, Planta Med. 70 (2004) 315-320.

[16] A.C. Luchini, P. Rodrigues-Orsi, S.H. Cestari, L.N. Seito, A. Witaicenis, C.H. Pellizzon, L.C. Di Stasi, Intestinal anti-inflammatory activity of coumarin and 4-hydroxycoumarin in the trinitrobenzenesulphonic acid model of rat colitis, Biol.
Pharm. Bull. 31 (2008) 1343-1350.

[17] A. Witaicenis, A.C. Luchini, C.A. Hiruma-Lima, S.L. Felisbino, L.A. Justulin, N. Garrido-Mesa, P. Utrilla, J. Gálvez, L.C. Di Stasi, Mechanism and effect of esculetin in an experimental animal model of inflammatory bowel disease, Eur. J. Inflamm. 11 (2013) 433-446.

[18] A. Witaicenis, L.N. Seito, A. da Silveira Chagas, L.D. de Almeida Jr., A.C. Luchini, P. Rodrigues-Orsi, S.H. Cestari, L.C. Di Stasi, Antioxidant and intestinal anti-inflammatory effects of plant-derived coumarin derivatives, Phytomedicine 21 (2014) $240-246$

[19] J.E. Krawisz, P. Sharon, W.F. Stenson, Quantitative assay for acute intestinal inflammation based on myeloperoxidase activity. Assessment of inflammation in rat and hamster models, Gastroenterology 87 (1984) 1344-1350.

[20] M.E. Anderson, Determination of glutathione and glutathione disulfide in biological samples, Methods Enzym. 113 (1985) 548-555.

[21] L.A. Dieleman, M.J. Palmen, H. Akol, E. Bloemena, A.S. Pena, S.G. Meuwissen, E.P. Van Rees, Chronic experimental colitis induced by dextran sulphate sodium (DSS) is characterized by Th1 and Th2 cytokines, Clin. Exp. Immunol. 114 (1998) 385-391.

[22] A.F. Stucchi, S. Shofer, S. Leeman, O. Materne, E. Beer, J. McClung, K. Shebani, F. Moore, M. O'Brien, J.M. Becker, NK-1 antagonist reduces colonic inflammation and oxidative stress in dextran sulfate-induced colitis in rats, Am. J. Physiol. Gastrointest. Liver Physiol. 279 (2000) G1298-G1306.

[23] M. Vicario, C. Amat, M. Rivero, M. Moreto, C. Pelegri, Dietary glutamine affects mucosal functions in rats with mild DSS-induced colitis, J. Nutr. 137 (2007) 1931-1937.

[24] S.M. Livingstone Solomon, Peter Mallon, Eilish Donnelly, Margaret Hoper, Morris Loughrey, Stephen Kirk, Keith Gardiner, The Dextran Sulphate Sodium (DSS) Model of Colitis: an Overview, Springer, 2014.

[25] J. Ni, S.F. Chen, D. Hollander, Effects of dextran sulphate sodium on intestinal epithelial cells and intestinal lymphocytes, Gut 39 (1996) 234-241.

[26] Y. Chen, K. Chou, E. Fuchs, W.L. Havran, R. Boismenu, Protection of the intestina mucosa by intraepithelial gamma delta T cells, Proc. Natl. Acad. Sci. U. S. A. 99 (2002) 14338-14343.

[27] C.R. Damiani, C.A. Benetton, C. Stoffel, K.C. Bardini, V.H. Cardoso, G. Di Giunta, R.A. Pinho, F. Dal-Pizzol, E.L. Streck, Oxidative stress and metabolism in animal model of colitis induced by dextran sulfate sodium, J. Gastroenterol. Hepatol. 22 (2007) 1846-1851.

[28] Oxidative stress in ulcerative colitis-associated carcinogenesis, Pathology, Res. Pract. 204 (2008) 511-524.

[29] M.J. Davies, Myeloperoxidase-derived oxidation: mechanisms of biological damage and its prevention, J. Clin. Biochem. Nutr. 48 (2011) 8-19.

[30] M.L. Circu, T.Y. Aw, Glutathione and modulation of cell apoptosis, Biochim. Biophys. Acta 1823 (2012) 1767-1777.

[31] H. Zhu, Y.R. Li, Oxidative stress and redox signaling mechanisms of inflammatory bowel disease: updated experimental and clinical evidence, Exp. Biol. Med. (Maywood) 237 (2012) 474-480.

[32] L.M. Kabeya, C.N. Fuzissaki, M.F. Andrade, A.E. Azzolini, S.H. Taleb-Contini, R.B. Vermelho, J.L. Lopes, Y.M. Lucisano-Valim, 4-methylcoumarin derivatives inhibit human neutrophil oxidative metabolism and elastase activity, J. Med. Food 16 (2013) 692-700.

[33] A.A. Geronikaki, A.M. Gavalas, Antioxidants and inflammatory disease: synthetic and natural antioxidants with anti-inflammatory activity, Comb. Chem. High. Throughput Screen 9 (2006) 425-442.

[34] T.T. Pizarro, F. Cominelli, Cytokine therapy for Crohn's disease: advances in translational research, Annu. Rev. Med. 58 (2007) 433-444.

[35] L. De Fazio, E. Cavazza, E. Spisni, A. Strillacci, M. Centanni, M. Candela, C. Pratico, M. Campieri, C. Ricci, M.C. Valerii, Longitudinal analysis of inflammation and microbiota dynamics in a model of mild chronic dextran sulfate sodium-induced colitis in mice, World J. Gastroenterol. 20 (2014) 2051-2061.

[36] P. Kiesler, I.J. Fuss, W. Strober, Experimental models of inflammatory bowel diseases, Cell Mol. Gastroenterol. Hepatol. 1 (2015) 154-170.

[37] W. Strober, I.J. Fuss, R.S. Blumberg, The immunology of mucosal models of inflammation, Annu. Rev. Immunol. 20 (2002) 495-549. 